\title{
Indications of Endoscopic Submucosal Dissection for Undifferentiated Early Gastric Cancer: Current Status and Future Perspectives for Further Expansion
}

\author{
Kazuo Shiotsuki $^{a}$ Kohei Takizawa $^{\mathrm{b}}$ Hiroyuki Ono $^{\mathrm{a}}$ \\ aDivision of Endoscopy, Shizuoka Cancer Center, Shizuoka, Japan; ${ }^{\text {bD }}$ epartment of Gastroenterology and \\ Endoscopy, Sapporo Kinentou Hospital, Hokkaido, Japan
}

\section{Keywords}

Endoscopic submucosal dissection - Early gastric cancer . Undifferentiated type - Lymph node metastasis .

Noncurative resection

\begin{abstract}
Background: Endoscopic submucosal dissection (ESD) is a widely accepted and minimally invasive treatment for early gastric cancer $(E G C)$ without the risk of lymph node metastasis (LNM). However, undifferentiated-type EGC (UD-EGC) is considered to have a relatively high risk of LNM. Recently, the Japan Clinical Oncology Group conducted a nonrandomized confirmatory trial (JCOG1009/1010) to evaluate the efficacy and safety of ESD for UD-EGC. Herein, we review the results of JCOG1009/1010 and the possibility of further expanding the indications for ESD. Summary: JCOG1009/1010 showed excellent technical results and 5-year overall survival in patients with UD-EGC. Based on the results, ESD for UD-EGC (cT1a) of $\leq 2 \mathrm{~cm}$ without ulceration was technically feasible and acceptable for standard treatment instead of gastrectomy with lymph node dissection. A review of the EGC of mixed histological type (mixed EGC) suggested that the mixed EGC might have worse biological behavior than the
\end{abstract}

karger@karger.com

(c) 2021 S. Karger AG, Basel

www.karger.com/dig

Karger" pure histological type. In cases of intramucosal EGC with pure signet-ring cell carcinoma or presenting a double-layer structure, the risk of LNM might be relatively low. Thus, there is a possibility of further expanding the indications or curative evaluations. In the case of UD-EGC after noncurative resection, the data suggest that the eCura system may be applicable to UD-EGC; however, due to the small number of cases, further study is warranted. Key Message: This review summarizes the present knowledge regarding indications for UD-EGC and the possibility of further expanding them.

(c) 2021 S. Karger AG, Basel

\section{Introduction}

Gastrectomy with lymph node (LN) dissection is a standard treatment for early gastric cancer (EGC) [1]. Gastrectomy with LN dissection can be invasive, and the loss of the stomach often results in a decreased quality of life. In contrast, endoscopic resection (ER) is a less-invasive treatment and has a better postoperative quality of life than gastrectomy because it preserves the stomach. Due to the possibility of en bloc resection and the risk of lymph node metastasis (LNM), the indications for ER 
have been limited to EGC diagnosed as differentiatedtype intramucosal cancer (cT1a) of $\leq 2 \mathrm{~cm}$ without ulceration [2].

Recently, endoscopic submucosal dissection (ESD) was developed and has been used worldwide [3-5]. It has made en bloc resection possible regardless of size and ulceration; therefore, the expansion of the indications for ESD is of interest.

Gotoda et al. [6] investigated the rate of LNM from surgically resected specimens of EGC to identify the conditions for extremely low possibility of LNM and proposed expanded indications for ER. Differentiatedtype EGC (cT1a) of $>2 \mathrm{~cm}$ without ulceration and differentiated-type EGC (cT1a) of $\leq 3 \mathrm{~cm}$ with ulceration were considered as possibly having negligible risk of LNM. In cases of undifferentiated-type EGC (UD-EGC), Hirasawa et al. [7] found that the risk of LNM in patients with UD-EGC (cT1a) of $\leq 2 \mathrm{~cm}$ without ulceration was negligible for LNM; therefore, these lesions were regarded as an expanded indication for ER. Subsequently, retrospective studies have reported that ESD for UD-EGC was efficacious and had favorable long-term outcomes [8-10]. To confirm the efficacy and safety of ESD for expanded indications of UD-EGC, a prospective multicenter clinical trial (Japan Clinical Oncology Group study, JCOG1009/1010) was conducted $[11,12]$. In this article, we review the results of JCOG1009/1010 and the possibility of further expanding the indications for ESD in UD-EGC.

\section{Expanded Indication of ESD for Undifferentiated- Type EGC}

To evaluate the safety and efficacy of ESD for UDEGC, a multi-institutional clinical trial (JCOG1009/1010) was performed [12]. The key inclusion criteria were endoscopically diagnosed cT1a/N0M0, $\leq 2 \mathrm{~cm}$ in size, without ulceration, with histologically proven components of undifferentiated carcinoma. The primary endpoint was 5-year overall survival (OS) in patients with UD-EGC. UD-EGC was defined as a diagnosis presenting undifferentiated carcinoma as the main histological type based on ESD specimens. A total of 346 patients were enrolled from 49 institutions in Japan between February 2011 and May 2013; 9 patients were considered ineligible. The median age was 62 years (range, $23-$ 80 years), and 179 (53\%) patients were men. In all eligible patients $(n=337)$, the median procedure time was 65 min (range, 12-459 min). En bloc resection was achieved in 332 (99\%) patients. Intraoperative perforation during ESD occurred in 13 patients (3.9\%), 12 of whom were successfully treated by endoscopic closure with clipping; 1 patient $(0.3 \%)$ required emergency surgery. Delayed perforation occurred in 6 patients $(1.8 \%)$, 5 of whom improved without additional treatment, while 1 patient underwent emergency surgery. ESD-related grade 4 adverse events were not observed. According to the pathological evaluation of the ESD specimens, 275 patients were diagnosed with UD-EGC; these were the subjects of the primary endpoint analysis. Among the 275 patients, the 5-year OS was $99.3 \%$ (95\% CI: $97.8-95.8 \%)$, and the 5-year relapse-free survival was $98.9 \%$ (95\% CI: 96.6-96.6\%). The 5-year OS in patients with UD-EGC exceeded the threshold value for 5 -year OS that was predetermined from the expected 5 -year OS of the general population adjusted for sex and age; therefore, the primary endpoint was met.

Based on the results of this study, ESD for UD-EGC (cT1a) of $\leq 2 \mathrm{~cm}$ without ulceration was technically feasible and acceptable for standard treatment instead of gastrectomy with LN dissection. In the latest guidelines in Japan [13], this category has been changed from expanded indications to absolute indications.

\section{Histological Behavior of EGC with Mixed Histological Type}

According to the Japanese gastric cancer treatment guidelines [14], histological evaluation of biopsy and ESD resection specimens is divided into differentiated and undifferentiated types. If both components are present, the quantitatively dominant component is defined as the main histological type. Although mixed histological types of EGC (mixed EGC) are often encountered in clinical practice, the Japanese gastric cancer guidelines do not address how to handle mixed EGC, and the histological behavior is incompletely understood.

To evaluate the risk of LNM in mixed EGC, Takizawa et al. [15] investigated the risk of LNM in mixed EGC from surgically resected cases. The histological types were classified into 4 categories according to the proportion of differentiated and undifferentiated adenocarcinoma components: pure differentiated type (PD), mixed predominantly differentiated type (MD), pure undifferentiated type (PU), and mixed predominantly undifferentiated type (MU). The definition of the mixed type was defined as MD if $>50 \%$ of the quantitatively dominant components are the differentiated type; if $>50 \%$ of the quantitatively dominant components are the undifferentiated type, it was defined as MU. The risk of LNM was higher in MD than in PD ( $11.1 \%$ vs. $3.1 \%)$, but the difference was not statistically 


\begin{tabular}{|c|c|c|c|c|c|c|c|}
\hline & \multirow{3}{*}{ [Ref. No] } & \multicolumn{6}{|c|}{ LNM, \% $(n / N)$} \\
\hline & & \multicolumn{2}{|c|}{ pT1a(M) } & \multicolumn{2}{|c|}{$\mathrm{pT} 1 \mathrm{a}(\mathrm{M})$ and $\mathrm{pUL0}$} & \multicolumn{2}{|c|}{$\mathrm{pT} 1 \mathrm{a}(\mathrm{M})$ and $\mathrm{pUL} 0$ and $\mathrm{pLVIC}$} \\
\hline & & $\leq 20 \mathrm{~mm}$ & $>20 \mathrm{~mm}$ & $\leq 20 \mathrm{~mm}$ & $>20 \mathrm{~mm}$ & $\leq 20 \mathrm{~mm}$ & $>20 \mathrm{~mm}$ \\
\hline Mixed EGC & [15] & \multicolumn{2}{|c|}{$14.6(14 / 96)$} & \multicolumn{2}{|c|}{$9.3(4 / 43)$} & \multicolumn{2}{|c|}{-} \\
\hline Pure UD & [18] & $1.7(3 / 178)$ & $3.9(13 / 336)$ & - & $3.6(3 / 84)$ & $0(0 / 66)$ & $2.4(2 / 82)$ \\
\hline SRC & [22] & \multicolumn{2}{|c|}{$6.2(16 / 258)$} & \multicolumn{2}{|c|}{-} & $0(0 / 77)$ & $1.7(3 / 174)$ \\
\hline DLS present & [26] & \multicolumn{2}{|c|}{$2.3(3 / 129)$} & \multicolumn{2}{|c|}{-} & - & $0(0 / 53)$ \\
\hline
\end{tabular}

Fig. 1. Summary of the risk of LNM in mixed EGC, pure-UD, SRC, and presenting DLS in intramucosal carcinoma. LNM, lymph node metastasis; EGC, early gastric cancer; SRC, signet-ring cell carcinoma; DLS, doublelayer structure; LVI, lymphovascular invasion.

significant. In comparison, the risk of LNM was significantly higher in MU than in PU (19.0\% vs. 6.0\%). These results suggest that the risk of LNM is different between the mixed and pure types.

Komatsu et al. [16] divided patients who underwent gastrectomy with LN dissection into mixed and nonmixed types to compare their long-term prognosis. The study showed that the 5-year disease-specific survival of patients with the mixed type was significantly worse than that of patients with the nonmixed type ( $96.5 \%$ vs. $99.5 \%)$ $(p<0.005)$. Furthermore, mixed EGC was independently associated with worse outcomes in multivariate logistic regression analysis.

Regarding ER cases, Horiuchi et al. [17] reported that mixed-type UD-EGC tended to undergo noncurative resection. Multivariate analysis of predictive factors for noncurative resection in UD-EGC identified the mixed type (odds ratio: OR 2.75, 95\% CI: 1.16-6.48). The rate of curative resection was significantly lower in the mixedtype UD-EGC than in pure signet-ring cell carcinoma (SRC) $(77.7 \%$ vs. $93.8 \%)$.

Thus far, we have reviewed the mixed histology and found that mixed EGC may have a higher risk of LNM, worse long-term prognosis, and worse biological behavior than the pure histological type of EGC. The current guidelines do not address mixed EGC, but it may be better to handle mixed EGC separately in some situations because of the worse biological behavior.
Histological Behavior of Signet-Ring Cell Carcinoma

It was suggested that mixed EGC is highly malignant, while pure UD-EGC is less malignant. Horiuchi et al. [18] investigated the risk of LNM among only UD-EGC; depth was limited to the intramucosal or submucosal layer from surgically resected cases. This study showed that LNM did not occur in patients with the pure undifferentiated type (PU), $\leq 40 \mathrm{~mm}$ in size, without ulceration, and without lymphovascular invasion. These results suggest that the risk of LNM for PU EGC in the intramucosal layer might be negligible. Several studies also have shown that the rate of LNM is relatively low in PU, especially in SRC $[19,20]$.

Hyung et al. [21] investigated the clinicopathological features of early SRC in surgical specimens. The study showed that SRC histology had a significantly lower rate of LNM (5.7\%) compared to non-SRC histology (16.0\%) $(p<0.001)$. Multivariate logistic regression analysis for LNM identified non-SRC histology as a risk factor. Moreover, the 5-year OS of patients with SRC was significantly higher than that of patients with non-SRC (94.2\% vs. $89.7 \%)(p=0.014)$. Ha et al. [22] also reported that SRC showed a lower rate of LNM (9.5\%) than non-SRC (24.9\%). That study showed that the 5-year disease-specific survival in patients with SRC was better than that in patients with non-SRC (99.7\% vs. $97.2 \%)(p=0.01)$. Based on these reports, it may be possible to further expand indications for ESD, especially in pure SRC, because of lower malignancy. 
Table 1. Patient and lesion characteristics

\begin{tabular}{|c|c|}
\hline & $\begin{array}{l}123 \text { patients } \\
123 \text { lesions }\end{array}$ \\
\hline Median age, years (range) & $67(38-85)$ \\
\hline \multicolumn{2}{|l|}{ Gender, n (\%) } \\
\hline Male & $81(66)$ \\
\hline Female & $42(34)$ \\
\hline \multicolumn{2}{|l|}{ Location, $n$ (\%) } \\
\hline Upper third & $25(20)$ \\
\hline Middle third & $70(40)$ \\
\hline Lower third & $28(22)$ \\
\hline Median tumor size, $\mathrm{mm}$ (range) & $25(8-75)$ \\
\hline \multicolumn{2}{|l|}{ Histology, $n(\%)$} \\
\hline Pure sig & $23(19)$ \\
\hline Mixed type & $100(81)$ \\
\hline \multicolumn{2}{|l|}{ Depth of invasion, $n$ (\%) } \\
\hline pT1a (M) & $66(53)$ \\
\hline pT1b (SM) & $57(47)$ \\
\hline \multicolumn{2}{|l|}{ UL, $n(\%)$} \\
\hline ULO & $99(81)$ \\
\hline UL1 & $24(19)$ \\
\hline \multicolumn{2}{|l|}{ Ly, $n(\%)$} \\
\hline Ly0 & $91(74)$ \\
\hline Ly1 & $32(26)$ \\
\hline \multicolumn{2}{|l|}{$\mathrm{V}, n(\%)$} \\
\hline V0 & $108(88)$ \\
\hline V1 & $15(12)$ \\
\hline \multicolumn{2}{|l|}{$\mathrm{HM}, n(\%)$} \\
\hline HMO & $118(96)$ \\
\hline HM1 & $5(4)$ \\
\hline \multicolumn{2}{|l|}{ VM, $n(\%)$} \\
\hline VMO & $114(93)$ \\
\hline VM1 & $9(7)$ \\
\hline
\end{tabular}

UL, ulceration; Ly, lymphatic invasion; V, venous invasion; HM, horizontal margin; VM, vertical margin.

\section{Double-Layer Structure in Signet-Ring Cell Carcinoma}

Sugihara et al. [23] reported that SRC often has a double-layer structure (DLS) in the intramucosal layer. The DLS consists of a superficial layer comprising intracytoplasmic mucin with an eccentric nucleus and a deep layer comprising poor intracytoplasmic mucin and acidophilic cytoplasm. Small round cells proliferate between the layers. Natsagdorji et al. [24] reported that the DLS displays functional differentiation to the normal gastric grand, and that the rate of submucosal invasion decreases if the DLS is maintained. Please see figure 1 in [25] for a typical case.

Takizawa et al. [26] investigated the relationship between the DLS and LNM of UD-EGC in surgical specimens. This study showed that absence of a DLS was a risk factor for submucosal invasion; if a DLS was present in the intramucosal lesions, there were no cases of LNM (0/69). They hypothesized that SRC that maintains a DLS within the intramucosa would not invade the submucosa and have a relatively low risk of LNM. Murai et al. [25] investigated the risk of LNM in SRC from surgically resected cases. In this study, 310 lesions of pathological intramucosal SRC were reviewed; LNM was present in 21 lesions, and 129 lesions (42\%) had a DLS present. Univariate analysis revealed that tumor size $>20 \mathrm{~mm}$, presence of ulceration, and absence of a DLS were significant risk factors for LNM. Multivariate logistic regression analysis identified only DLS as a risk factor for LNM (OR, 4.93; 95\% CI: 1.41-17.3) ( $p=0.01)$. In this study, among tumor sizes $>20 \mathrm{~mm}$ with no lymphovascular invasion, a DLS was present, and there were no cases of LNM (0/53). Therefore, they suggested that immunohistochemical staining should be performed in cases with an SRC $>20 \mathrm{~mm}$; if a DLS is present, gastrectomy with LN dissection may not be necessary due to the relatively low risk of LNM. In the future, the presence or absence of a DLS may be included in the evaluation criteria for curability. So far, we have summarized the risk of LNM in mixed EGC, pure UD, SRC, and presenting DLS (shown in Fig. 1).

\section{LNM in Patients with Additional Gastrectomy after ESD for Undifferentiated-Type EGC with Noncurative} Resection

As we mentioned above, the expanded indications for ESD for differentiated-type EGC and UD-EGC were changed to absolute indications, based on the positive results of JCOG0607 [27] and JCOG1009/1010 [12]. In each of these studies, noncurative resections were observed in $>20 \%$ of cases. According to the Japanese gastric cancer treatment guidelines, radical gastrectomy with LN dissection is recommended for noncurative resections [14]. However, despite performing radical surgery, the rate of LNM is relatively low $[28,29]$, and risk stratification of noncurative resections is desired.

In Japan, the Establishment of Accommodation of Early Stomach Cancer Treatment (EAST) study group conducted a multicenter retrospective study to determine the risk of LNM after noncurative resection. Hatta et al. [30] reported the risk of LNM and risk stratification systems after noncurative resection. Multivariate logistic regression analysis showed that independent risk factors for LNM were tumor size $>30 \mathrm{~mm}$ (OR, 2.03; 95\% CI: $1.28-$ 3.14), tumor depth (OR, 1.68; 95\% CI: 0.97-2.92), lymphatic invasion (OR, 3.99; 95\% CI: 2.43-6.55), venous invasion (OR, 1.65; 95\% CI: 1.01-2.70), and positive VM (OR, 1.81; 95\% CI: 1.10-3.00). From these results, they established a scoring system named the "eCura system," 
Table 2. Comparing our data with the data from the EAST study (analysis for the risk of LNM)

\begin{tabular}{|c|c|c|c|c|c|c|c|}
\hline & \multicolumn{3}{|c|}{ This study (univariate analysis) } & & \multicolumn{3}{|c|}{ EAST study (multivariate analysis) } \\
\hline & OR & $95 \% \mathrm{Cl}$ & $p$ value & & OR & $95 \% \mathrm{Cl}$ & $p$ value \\
\hline \multicolumn{8}{|l|}{ Ly } \\
\hline Positive & 4.27 & $1.29-14.1$ & 0.02 & & 3.99 & $2.43-6.55$ & $<0.01$ \\
\hline Negative & 1 & Reference & & & 1 & Reference & \\
\hline \multicolumn{8}{|l|}{$\mathrm{V}$} \\
\hline Positive & 10.8 & $3.44-33.9$ & $<0.01$ & & 1.65 & $1.01-2.70$ & 0.04 \\
\hline Negative & 1 & Reference & & & 1 & Reference & \\
\hline \multicolumn{8}{|l|}{ UL } \\
\hline Presence & 1.03 & $0.23-4.58$ & 1.00 & & 0.98 & $0.57-1.69$ & 1.00 \\
\hline Absence & 1 & Reference & & & 1 & Reference & \\
\hline \multicolumn{8}{|l|}{ VM } \\
\hline Positive & 3.17 & $0.79-12.8$ & 0.16 & & 1.81 & $1.10-3.00$ & 0.02 \\
\hline Negative & 1 & Reference & & & 1 & Reference & \\
\hline \multicolumn{8}{|l|}{ Size } \\
\hline$\geq 40 \mathrm{~mm}$ & 0.89 & $0.20-3.93$ & 1.00 & $>30 \mathrm{~mm}$ & 2.03 & $1.28-3.14$ & $<0.01$ \\
\hline$<40 \mathrm{~mm}$ & 1 & Reference & & $\leq 30 \mathrm{~mm}$ & 1 & Reference & \\
\hline \multicolumn{8}{|l|}{ Depth } \\
\hline SM & 4.63 & $1.03-20.9$ & 0.04 & SM2 & 1.68 & $0.97-2.92$ & 0.07 \\
\hline$M$ & 1 & Reference & & M/SM1 & 1 & Reference & \\
\hline
\end{tabular}

LNM, lymph node metastasis; OR, odds ratio.

which assigns a risk score based on these factors and can predict the risk of LNM. Risk of LNM can be classified into 3 categories: low risk ( $0-1$ point), intermediate risk (2-4 points), and high risk (5-7 points) with LNM rates of $2.5 \%, 6.7 \%$, and $22.7 \%$, respectively. While it is a wellvalidated scoring system, $>80 \%$ of the cases in the development and validation cohorts consisted of differentiated-type EGC. Thus, it is not clear whether these results are applicable to UD-EGC after noncurative resection.

Therefore, to evaluate the LNM of UD-EGC after noncurative resection, and whether the eCura system could be applied for UD-EGC, we reviewed a chart-based investigation of 146 consecutive patients with UD-EGC after noncurative resection who underwent additional gastrectomy with LN dissection at the Shizuoka Cancer Center from September 2002 to December 2019. This study was approved by the Ethics Committee of the Shizuoka Cancer Center (Approval No. J2021-80). The exclusion criteria were (1) synchronous EGC that did not meet the curative criteria of ESD, (2) ESD in the remnant stomach or gastric tube, (3) additional treatment with photodynamic therapy, (4) invasion of the muscularis propria or deeper in the pathology of the surgical or endoscopic specimen, and (5) missing data. During the study period, 146 patients met the inclusion criteria, but 23 patients were excluded: 8 patients had synchronous EGC that did not meet the curative criteria, 7 patients had ESD in the remnant stomach or gastric tube, 3 patients received photodynamic therapy, 4 cases had EGC invasion to the muscularis propria or deeper, and 1 case had missing data. A total of 123 patients were analyzed.

The median age was 67 years (range, $38-85$ years), and $81(66 \%)$ patients were men. The median tumor size was 25 $\mathrm{mm}$ (range, $8-75 \mathrm{~mm}$ ). The histological types included 23 pure SRC (19\%) and 100 mixed-type UD-EGC (81\%). In addition, ulceration was present in 24 lesions (20\%), lymphatic invasion was present in 32 lesions (26\%), and venous invasion was present in 15 lesions (12\%). The vertical margin was positive in 9 cases (7\%), and the horizontal margin was positive in 5 cases (4\%) (Table 1). LNM was observed in 10 patients $(8.1 \%)$. In univariate analyses, lymphatic invasion (OR, 4.27; 95\% CI: $1.29-14.1)(p=0.02)$, venous invasion (OR, 10.8; 95\% CI: 3.44-33.9) $(p<0.01)$, and submucosal invasion (OR, 4.63; 95\% CI: 1.03-20.9) $(p=0.04)$ were independent risk factors for LNM. Multivariate logistic analysis was not performed because of the small number of cases.

Comparison of our data with the data from the EAST study [30] (Table 2) showed a similar risk of LNM, but the ORs were slightly different. Our study suggests that the eCura system may be applicable to UD-EGC, but due to the small number of cases, we are planning a multicenter study to confirm the results.
Shiotsuki/Takizawa/Ono 


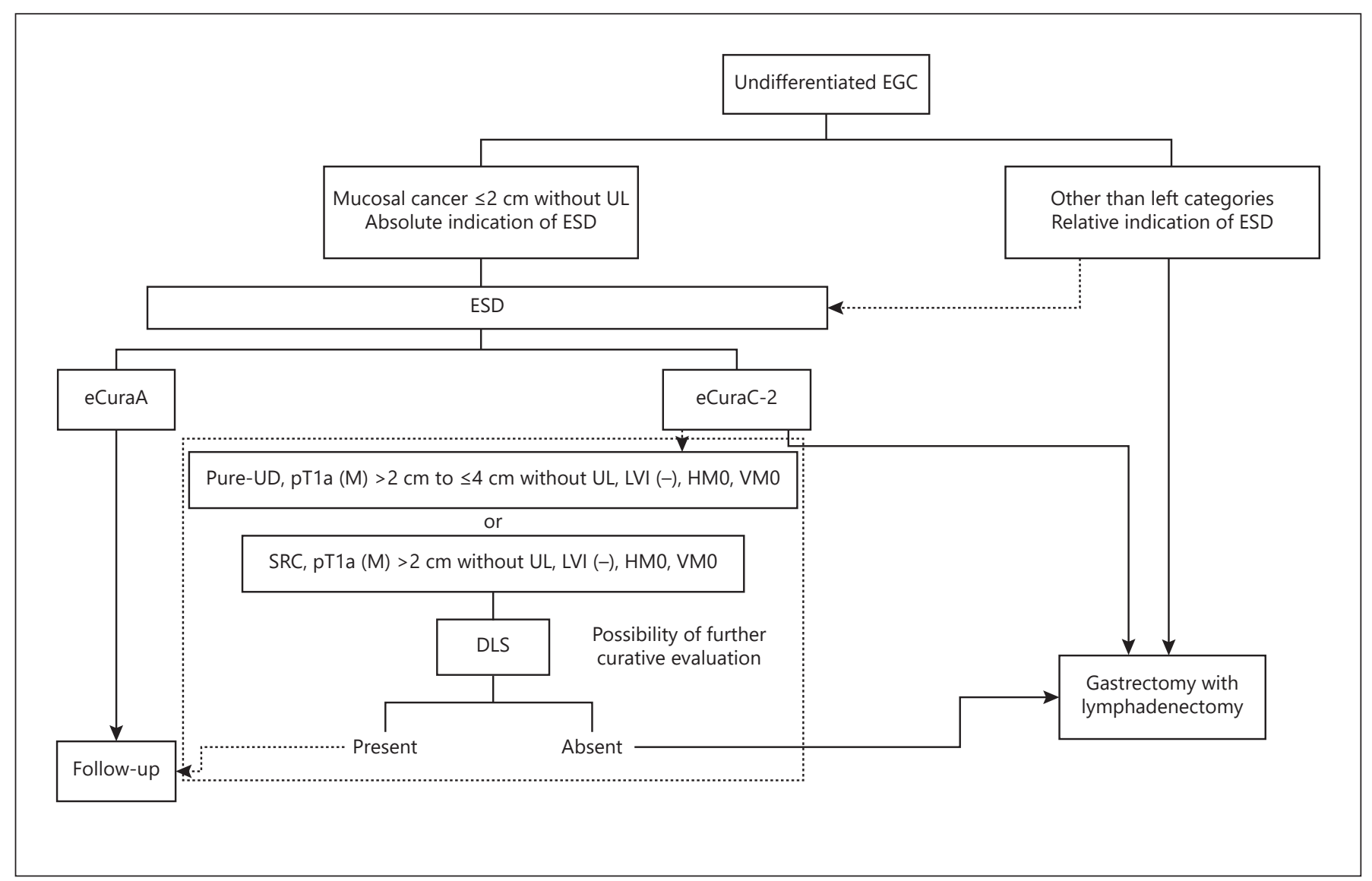

Fig. 2. Future perspectives in the treatment strategy for undifferentiated EGC. EGC, early gastric cancer; ESD, endoscopic submucosal dissection; DLS, double-layer structure.

\section{Conclusion}

According to the positive results of a multicenter prospective study (JCOG1009/1010), expanded indications of ESD for UD-EGC (cT1a) of $\leq 2 \mathrm{~cm}$ in size without ulceration have become an absolute indication in the newest guidelines in Japan. In patients with histology of pure SRC or presenting a DLS, the risk of LNM might be relatively low; therefore, there is a possibility of further expansion of indications or curative evaluation (shown in Fig. 2). It is not clear whether the eCura system can be applicable to UD-EGC after noncurative resection, so further study is warranted.

\section{Acknowledgments}

We express our sincere gratitude to the medical staff members in our endoscopy unit for their collaboration on this work.

\section{Conflict of Interest Statement}

The authors declare that they have no conflicts of interest related to this study.

\section{Funding Sources}

This work did not receive any funding.

\section{Author Contributions}

Kazuo Shiotsuki had full access to all data in the study and took responsibility for the integrity of the data and the accuracy of the data analysis. Kazuo Shiotsuki and Kohei Takizawa drafted the manuscript. 


\section{References}

1 Japanese Gastric Cancer Association Registration Committee, Maruyama K, Kaminishi M, Hayashi K, Isobe Y, Honda I, et al. Gastric cancer treated in 1991 in Japan: data analysis of nationwide registry. Gastric Cancer. 2006;9(2): 51-66.

2 Japanese Gastric Cancer Association. Japanese gastric cancer treatment guidelines 2010 (ver. 3). Gastric Cancer. 2011;14(2):113-23.

3 Ono H, Kondo H, Gotoda T, Shirao K, Yamaguchi H, Saito D, et al. Endoscopic mucosal resection for treatment of early gastric cancer. Gut. 2001;48(2):225-9.

4 Oda I, Gotoda T, Hamanaka H, Eguchi T, Saito Y, Matsuda T, et al. Endoscopic submucosal dissection for early gastric cancer: technical feasibility, operation time and complications from a large consecutive series. Dig Endosc. 2005; 17(1):54-8.

5 Ono $\mathrm{H}$, Hasuike N, Inui T, Takizawa K, Ikehara $\mathrm{H}$, Yamaguchi $\mathrm{Y}$, et al. Usefulness of a novel electrosurgical knife, the insulation-tipped diathermic knife-2, for endoscopic submucosal dissection of early gastric cancer. Gastric Cancer. 2008;11(1):47-52.

6 Gotoda T, Yanagisawa A, Sasako M, Ono H, Nakanishi Y, Shimoda T, et al. Incidence of lymph node metastasis from early gastric cancer: estimation with a large number of cases at two large centers. Gastric Cancer. 2000;3(4): 219-25.

7 Hirasawa T, Gotoda T, Miyata S, Kato Y, Shimoda $\mathrm{T}$, Taniguchi $\mathrm{H}$, et al. Incidence of lymph node metastasis and the feasibility of endoscopic resection for undifferentiated-type early gastric cancer. Gastric Cancer. 2009;12(3):148-52.

8 Yamamoto Y, Fujisaki J, Hirasawa T, Ishiyama A, Yoshimoto K, Ueki N, et al. Therapeutic outcomes of endoscopic submucosal dissection of undifferentiated-type intramucosal gastric cancer without ulceration and preoperatively diagnosed as 20 millimetres or less in diameter. Dig Endosc. 2010;22(2):112-8.

9 Okada K, Fujisaki J, Yoshida T, Ishikawa H, Suganuma T, Kasuga A, et al. Long-term outcomes of endoscopic submucosal dissection for undifferentiated-type early gastric cancer. Endoscopy. 2012;44:122-7.

10 Abe S, Oda I, Suzuki H, Nonaka S, Yoshinaga $\mathrm{S}$, Odagaki T, et al. Short- and long-term outcomes of endoscopic submucosal dissection for undifferentiated early gastric cancer. Endoscopy. 2013;45(9):703-7.

11 Takizawa K, Takashima A, Kimura A, Mizusawa J, Hasuike N, Ono H, et al. A phase II clinical trial of endoscopic submucosal dissection for early gastric cancer of undifferentiated type: Japan Clinical Oncology Group study JCOG1009/1010. Jpn J Clin Oncol. 2013;43(1): 87-91.

12 Takizawa K, Ono H, Hasuike N, Takashima A, Minashi K, Boku N, et al. A non-randomized, single-arm confirmatory trial of expanded endoscopic submucosal dissection indication for undifferentiated early gastric cancer: Japan Clinical Oncology Group study (JCOG1009/1010). Gastric Cancer. 2021 Mar; 24(2):479-91.

13 Ono H, Yao K, Fujishiro M, Oda I, Uedo N, Nimura S, et al. Guidelines for endoscopic submucosal dissection and endoscopic mucosal resection for early gastric cancer (second edition). Dig Endosc. 2021 Jan;33(1):4-20.

14 Japanese Gastric Cancer Association. Japanese gastric cancer treatment guidelines 2018, ed 5. Gastric Cancer. 2021;24:1-21.

15 Takizawa K, Ono H, Kakushima N, Tanaka M, Hasuike N, Matsubayashi $\mathrm{H}$, et al. Risk of lymph node metastases from intramucosal gastric cancer in relation to histological types: how to manage the mixed histological type for endoscopic submucosal dissection. Gastric Cancer. 2013;16(4):531-6.

16 Komatsu S, Ichikawa D, Miyamae M, Shimizu $\mathrm{H}$, Konishi H, Shiozaki A, et al. Histological mixed-type as an independent prognostic factor in Stage I gastric carcinoma. World J Gastroenterol. 2015;21(2):549-55.

17 Horiuchi Y, Fujisaki J, Yamamoto N, Ishizuka $\mathrm{N}$, Omae $\mathrm{M}$, Ishiyama $\mathrm{A}$, et al. Mixed poorly differentiated adenocarcinoma in undifferentiated-type early gastric cancer predicts endoscopic noncurative resection. Gastric Cancer. 2018;21(4):689-95.

18 Horiuchi Y, Ida S, Yamamoto N, Nunobe S, Ishizuka N, Yoshimizu S, et al. Feasibility of further expansion of the indications for endoscopic submucosal dissection in undifferentiatedtype early gastric cancer. Gastric Cancer. 2020; 23(2):285-92.

19 Lee SH, Jee SR, Kim JH, Seol SY. Intramucosal gastric cancer: the rate of lymph node metastasis in signet ring cell carcinoma is as low as that in well-differentiated adenocarcinoma. Eur J Gastroenterol Hepatol. 2015;27(2):170-4.

20 Kim HM, Pak KH, Chung MJ, Cho JH, Hyung WJ, Noh SH, et al. Early gastric cancer of signet ring cell carcinoma is more amenable to endoscopic treatment than is early gastric cancer of poorly differentiated tubular adenocarcinoma in select tumor conditions. Surg Endosc. 2011; 25(9):3087-93.
21 Hyung WJ, Noh SH, Lee JH, Huh JJ, Lah KH, $\mathrm{Choi} \mathrm{SH}$, et al. Early gastric carcinoma with signet ring cell histology. Cancer. 2002;94(1):7883.

22 Ha TK, An JY, Youn HK, Noh JH, Sohn TS, Kim S. Indication for endoscopic mucosal resection in early signet ring cell gastric cancer. Ann Surg Oncol. 2008;15(2):508-13.

23 Sugihara H, Hattori T, Fukuda M, Fujita S. Cell proliferation and differentiation in intramucosal and advanced signet ring cell carcinomas of the human stomach. Virchows Arch A Pathol Anat Histopathol. 1987;411(2):117-27.

24 Natsagdorj L, Sugihara H, Bamba M, Hattori T. Intratumoural heterogeneity of intestinal expression reflects environmental induction and progression-related loss of induction in undifferentiated-type gastric carcinomas. Histopathology. 2008;53(6):685-97.

25 Murai K, Takizawa K, Shimoda T, Fujii S, Sugino T, Yoshida $M$, et al. Effect of double-layer structure in intramucosal gastric signet-ring cell carcinoma on lymph node metastasis: a retrospective, single-center study. Gastric Cancer. 2019;22(4):751-8.

26 Takizawa K, Shimoda T, Nakanishi Y, Taniguchi H, Oda I, Gotoda T, et al. Expanded indication for endoscopic resection from pathological viewpoint: the possibility of sm invasion by undifferentiated-type early gastric cancer. Stomach Intest. 2006;41:9-17. Japanese.

27 Hasuike N, Ono H, Boku N, Mizusawa J, Takizawa K, Fukuda H, et al. A non-randomized confirmatory trial of an expanded indication for endoscopic submucosal dissection for intestinal-type gastric cancer (cT1a): the Japan Clinical Oncology Group study (JCOG0607). Gastric Cancer. 2018;21(1):114-23.

28 Suzuki H, Oda I, Abe S, Sekiguchi M, Nonaka S, Yoshinaga S, et al. Clinical outcomes of early gastric cancer patients after noncurative endoscopic submucosal dissection in a large consecutive patient series. Gastric Cancer. 2017;20(4): 679-89.

29 Kawata N, Kakushima N, Takizawa K, Tanaka M, Makuuchi R, Tokunaga M, et al. Risk factors for lymph node metastasis and long-term outcomes of patients with early gastric cancer after non-curative endoscopic submucosal dissection. Surg Endosc. 2017;31(4):1607-16.

30 Hatta W, Gotoda T, Oyama T, Kawata N, Takahashi A, Yoshifuku Y, et al. A scoring system to stratify curability after endoscopic submucosal dissection for early gastric cancer: "eCura system". Am J Gastroenterol. 2017; 112(6):874-81. 